\title{
Gadolinium-Enhanced 3D T1-Weighted Black-Blood MR Imaging for the Detection of Acute Optic Neuritis
}

\author{
(D). Riederer, (D) N. Sollmann, (D) M. Mühlau, (D) C. Zimmer, and (D).S. Kirschke
}

\begin{abstract}
BACKGROUND AND PURPOSE: A 3D T1-weighted black-blood sequence was recently shown to improve the detection of contrastenhancing lesions in the brain in patients with MS compared with a 3D T1-weighted MPRAGE sequence. We compared a contrastenhanced 3D T1-weighted black-blood sequence with a dedicated orbital contrast-enhanced T1-weighted Dixon sequence in patients with acute optic neuritis.
\end{abstract}

MATERIALS AND METHODS: MR imaging data (3T) of 51 patients showing symptoms of acute optic neuritis were analyzed retrospectively, including whole-brain contrast-enhanced 3D T-weighted black-blood and dedicated orbital coronal 2D or 3D contrast-enhanced TT-weighted Dixon sequences. Two neuroradiologists assessed the images for overall image quality, artifacts, diagnostic confidence, and visual contrast enhancement. Furthermore, the standardized contrast-to-noise ratio was calculated. The final diagnosis of acute optic neuritis was established on the basis of clinical presentation, visually evoked potentials, and optical coherence tomography.

RESULTS: Thirty of 51 patients were diagnosed with acute optic neuritis. Of those, 21 showed contrast-enhancing lesions in the optic nerves, similarly detectable on contrast-enhanced T1-weighted Dixon and contrast-enhanced T1-weighted black-blood images. Thus, the accuracy for each sequence was identical, with a resulting sensitivity of $70 \%$ and specificity of $90 \%$ or $100 \%$ (depending on the reader). Overall image quality, diagnostic confidence, visual contrast enhancement, and artifacts were rated similarly in contrast-enhanced 3D T1-weighted black-blood and dedicated orbital contrast-enhanced T1-weighted Dixon sequences. There was no significant difference $(P=.27)$ in the mean standardized contrast-to-noise ratio between contrast-enhanced T1-weighted blackblood $(1.76 \pm 1.07)$ and contrast-enhanced T1-weighted Dixon $(2.29 \pm 2.49)$ sequences.

CONCLUSIONS: Contrast-enhanced 3D T1-weighted black-blood imaging is comparable in accuracy and qualitative/quantitative features with dedicated orbital contrast-enhanced T1-weighted Dixon imaging for the detection of acute optic neuritis. Therefore, when used, it has the potential to considerably shorten total patient imaging time.

ABBREVIATIONS: $\mathrm{BB}=$ black-blood; DIR = double inversion recovery; FS = fat-suppressed; OCT = optical coherence tomography; ON =optic neuritis; $\mathrm{VEP}=$ visually evoked potentials

$A$ cute optic neuritis (ON) may result in visual disturbances, pain during eye movement, and dyschromatopsia. Acute ON can occur as an isolated syndrome or can be associated with MS. In particular, patients with ON show a high risk of conversion to definite MS (34\%-75\%), ${ }^{1,2}$ and a high percentage of patients with MS develop ON during the course of the disease (70\%). ${ }^{3-5}$ Although discussed and recommended by the Magnetic Resonance Imaging in

Received February 7, 2020; accepted after revision July 29

From the Departments of Neuroradiology (I.R., N.S., C.Z., J.S.K.) and Neurology (M.M.), and Neuroimaging Center TUM-NIC, Klinikum rechts der Isar (M.M. and N.S.), School of Medicine, Technical University of Munich, Munich, Germany.

Paper previously presented, in part, at: Annual Meeting of the European Society for Neuroradiology, September 19-20, Oslo, Norway, and Annual Meeting of the German Society for Neuroradiology, October 9-10, Frankfurt, Germany, as oral presentations, and Annual Meeting of the European Committee for Treatment and Research in Multiple Sclerosis, September 11-13, 2019, Stockholm, Sweden as a poster.
MS (MAGNIMS; https://www.magnims.eu/) consensus guidelines on MS criteria in MR imaging, ${ }^{6} \mathrm{ON}$ has not yet been included in the criterion of dissemination in space in the McDonald criteria (2017) for the diagnosis of MS. ${ }^{7}$ Nevertheless, the importance of the involvement of the optic nerve was emphasized by the International Panel on MS Diagnosis, and further research on this issue was encouraged. ${ }^{6}$

Acute $\mathrm{ON}$ is traditionally diagnosed clinically by visually evoked potentials (VEP) and optical coherence tomography (OCT) and can

Please address correspondence to Isabelle Riederer, MD, Department of Diagnostic and Interventional Neuroradiology, Klinikum rechts der Isar, School of Medicine, Technical University of Munich, Ismaninger Str 22, 81675 Munich, Germany; e-mail: isabelle.riederer@tum.de

- Indicates open access to non-subscribers at www.ajnr.org http://dx.doi.org/10.3174/ajnr.A6807

AJNR Am J Neuroradiol 41:2333-38 Dec 2020 www.ajnr.org 
be diagnosed through MR imaging. The recommended MR imaging protocol for ON consists of coronal fat-suppressed (FS) T2WI. ${ }^{8}$ Recent studies have demonstrated that double inversion recovery (DIR) images can be used for the detection of ON with high sensitivity and specificity. ${ }^{9,10}$ Additionally, it has been shown that DIR images reveal acute and chronic optic nerve lesions with higher sensitivity compared with VEP. ${ }^{11}$ Coronal T1-weighted images before and after contrast administration are recommended for the detection of acute $\mathrm{ON}{ }^{8}$ Contrast enhancement can be detected in up to $94 \%$ of the patients with acute $\mathrm{ON}$, but results vary depending on study inclusion criteria and sequences used. ${ }^{8,12}$ Recently, a study showed that 3D T1weighted TSE black-blood (BB) images improve the detection rate of contrast-enhancing lesions in the brain in patients with $\mathrm{MS}^{13}$ or intracranial metastases ${ }^{14-17}$ compared with a conventional 3D T1-weighted MPRAGE sequence.

Originally, T1-weighted $\mathrm{BB}$ sequences had been designed to detect inflammatory changes of the vascular wall of the heart or blood vessels by distinct suppression of the blood signal. ${ }^{18,19}$ For this purpose, T1-weighted BB imaging was based on the DIR technique and initially restricted to $2 \mathrm{D}$ acquisition mode with only a small FOV coverage. Recently, a new type of T1-weighted BB TSE sequence was introduced with variable flip angle refocusing pulses, thus allowing 3D imaging with high isotropic resolution. ${ }^{17,20}$ This new T1-weighted BB sequence combines a highly sensitive depiction of contrast enhancement with fast imaging and high spatial resolution and is thus gaining popularity in routine MR imaging for different purposes. As a TSE-based approach, this sequence has fewer artifacts compared with gradient-echo-based sequences. The benefits of reduced artifacts, particularly at the skull base and the sensitive depiction of contrast enhancement may render T1weighted BB imaging well-suited for orbital imaging. In fact, a recent study showed that T1-weighted $\mathrm{BB}$ imaging is able to detect arteritic posterior ciliary artery involvement in patients with arteritic anterior ischemic optic neuropathy. ${ }^{21}$ However, T1-weighted $\mathrm{BB}$ imaging has not yet been assessed for acute $\mathrm{ON}$.

Here, we hypothesize that contrast-enhanced 3D T1-weighted BB imaging is superior to dedicated orbital FS contrast-enhanced T1WI for detecting acute $\mathrm{ON}$.

\section{MATERIALS AND METHODS \\ Patients}

Data were analyzed retrospectively with approval of the local ethics committee. The patient group consisted of 51 consecutive patients (31 women, 20 men; mean age, $35 \pm 11$ years; age range,
18-66 years) showing symptoms of acute ON (such as visual disturbances or pain during eye movement) who had undergone MR imaging between April 2018 and January 2020. All MR imaging data included dedicated orbital Dixon sequences and 3D T1weighted BB. None of them had to be excluded due to motion artifacts. Final diagnoses were established by the treating neurologists considering history, symptoms, response to steroid treatment, MR imaging findings, and paraclinical tests such as VEP or OCT.

\section{MR Imaging Acquisition}

MR imaging was performed on a 3T scanner (Ingenia; Philips Healthcare) using a 32-channel array head coil. MR imaging included 3D FLAIR, 3D DIR, coronal FS 2D T2-weighted sequences (T2 Dixon or T2 spectral presaturation with inversion recovery), contrast-enhanced 3D T1-weighted MPRAGE, and contrastenhanced 3D T1-weighted BB sequences of the whole brain in all patients, as well as coronal FS contrast-enhanced 2D T1-weighted Dixon $(n=43)$ or contrast-enhanced 3D T1-weighted Dixon $(n=$ 8 ) sequences covering the orbit. The acquisition parameters of T1weighted $\mathrm{BB}$ and $\mathrm{T} 1$-weighted Dixon sequences are shown in Table 1.

Due to the small number of 3D T1-weighted Dixon images $(n=$ 8), we pooled 2D and 3D T1-weighted Dixon images for the analysis. On average, contrast-enhanced T1-weighted Dixon was acquired about 5 minutes later than contrast-enhanced T1-weighted BB (T1-weighted BB was performed at a mean of 13:25 \pm 4:45 (min:sec) and contrast-enhanced T1-weighted BB, 8:11 \pm 4:07 (min:sec) after the intravenous administration of gadolinium).

\section{MR Imaging Analysis}

Imaging data were independently assessed by 2 neuroradiologists (blinded to the diagnosis and side of the affected optic nerve) on a standard PACS workstation. The images were read twice, first by assessing contrast-enhanced T1-weighted $\mathrm{BB}$ and excluding contrast-enhanced T1-weighted Dixon images and second by assessing contrast-enhanced T1-weighted Dixon and excluding contrast-enhanced T1-weighted BB with an interval of 2 weeks between both reads to avoid memory bias.

During each reading process, DIR and FS T2-weighted images could be consulted simultaneously. In case of 3D images, 1-mm-section-thickness reconstructions were performed in 3D in orientations perpendicular to and along the optic nerves, with sagittal reconstructions being generated in an oblique direction per side.

Table 1: Acquisition parameters of the T1-weighted sequences

\begin{tabular}{|c|c|c|c|}
\hline Sequence & T1-Weighted BB & T1-Weighted Dixon & T1-Weighted Dixon \\
\hline Sequence type & TSE & TFE & TSE \\
\hline Acquisition mode & $3 D$ & $3 \mathrm{D}$ & $2 \mathrm{D}$ \\
\hline Acquisition plane & Sagittal & Axial & Coronal \\
\hline Acquired voxel size $\left(\mathrm{mm}^{3}\right)$ & $0.90 \times 0.89 \times 0.90$ & $0.90 \times 0.99 \times 0.90$ & $0.60 \times 0.72 \times 2.5$ \\
\hline Acquisition matrix & $280 \times 281$ & $132 \times 157$ & $200 \times 210$ \\
\hline FOV (mm) & 240 & 154 & 151 \\
\hline TR (ms) & 700 & 6.2 & 500 \\
\hline TE (ms) & 35 & 0 & 9.8 \\
\hline Acquisition time (min) & 1:36 & 3:11 & $2: 00$ \\
\hline Average delay after IV administration of gadolinium (min:sec) & $8: 42$ & $12: 46$ & 13:36 \\
\hline
\end{tabular}


Both readers individually counted contrast-enhancing lesions in the optic nerves and assessed the images on a 5-point Likert scale for overall image quality in the orbit (1, nondiagnostic; 2 , poor; 3 , average; 4 , good; or 5 , excellent), artifacts (1, severe; 2 , pronounced; 3 , moderate; 4 , mild; or 5 , none), and diagnostic confidence (1, unlikely; 2 , vague; 3 , likely; 4 , high; or 5 , very high). Moreover, a 4-point Likert scale was considered for visual contrast enhancement of the optic nerves, if any (1, none; 2 , minimal; 3 , moderate; or 4, strong). Afterward, a consensus read was performed in patients with discrepant numbers of contrast-enhancing lesions. A contrast-enhancing lesion was defined as a circumscribed hyperintense signal within the optic nerve depicted after gadolinium administration on T1-weighted images.

In addition, 1 neuroradiologist categorized the localization of lesions into the following sections of the optic nerve: intraorbital, extraorbital intracanalicular, and extraorbital intracranial (chiasmatic) segments. Furthermore, a standardized contrast-to-noise ratio (sCNR) was measured. Because it is difficult to compare contrast-to-noise ratios between different sequences, we defined a "standardized" contrast-to-noise ratio as previously described ${ }^{22,23}$ by respecting the dependency of the contrast-to-noise ratio on voxel size and acquisition time of the sequences according to the following formula:

$\mathrm{sCNR}=\left(\mathrm{S}_{\text {lesion }}-\mathrm{S}_{\text {optic nerve }}\right) /\left(\mathrm{SD}_{\text {optic nerve }} \times \mathrm{V}_{\text {Voxel }} \times \sqrt{\mathrm{t}_{\text {acq }}}\right)$, where $S_{\text {lesion }}$ represents the mean signal in an ROI $\left(2-5 \mathrm{~mm}^{2}\right)$ in a contrast-enhancing lesion in the optic nerve, and $S_{\text {optic nerve }}$ represents the signal of an ROI in the contralateral optic nerve in a corresponding normal-appearing location. $\mathrm{SD}_{\text {optic nerve }}$ is the $\mathrm{SD}$ in a region in the nonaffected optic nerve, $\mathrm{V}_{\text {Voxel }}$ is the voxel size, and $\mathrm{t}_{\mathrm{acq}}$ represents the acquisition time of the sequence in seconds.

\section{Statistical Analyses}

Statistical analyses were performed using SPSS 25 (SPSS Statistics for Windows; IBM). Wilcoxon signed-rank tests were performed to compare overall image quality, artifacts, diagnostic confidence,

Table 2: Sensitivity and specificity

\begin{tabular}{ccc}
\hline $\begin{array}{c}\text { Final Diagnosis } \\
\text { "Acute ON"a }\end{array}$ & $\begin{array}{c}\text { CE + (Reader 1/ } \\
\text { Reader 2) }\end{array}$ & $\begin{array}{c}\text { CE - (Reader 1/ } \\
\text { Reader 2) }\end{array}$ \\
\hline ON+ & $21 / 21$ & $9 / 9$ \\
ON- & $0 / 2$ & $21 / 19$ \\
\hline
\end{tabular}

Note:-CE + indicates contrast enhancement; $C E-$, no contrast enhancement; $\mathrm{ON}+$, optic neuritis; $\mathrm{ON}-$, no optic neuritis.

${ }^{a}$ Reader 1: sensitivity, $21 / 30=70 \%$; specificity, $21 / 21=100 \%$; reader 2: sensitivity, $21 / 30=70 \%$; specificity, $19 / 21=90 \%$.

Table 3: Contingency table of all available diagnostics and the correlation of their results to contrast-enhancing lesions in MR imaging

\begin{tabular}{llcccc}
\hline & & & \multicolumn{2}{c}{ Other } \\
& Diagnosis & \multicolumn{2}{c}{ ON } & \multicolumn{3}{c}{ Diagnoses } \\
\hline Diagnostics (availability) & & CE + & CE- & CE+ & CE- \\
VEP (37/51) & + & 11 & 3 & 0 & 0 \\
& - & 1 & 3 & 0 & 19 \\
OCT (31/51) & + (Acute) & 3 & 2 & 0 & 0 \\
& + (Chronic) & 4 & 2 & 0 & 1 \\
& - & 4 & 4 & 0 & 11 \\
\hline
\end{tabular}

Note:-CE + indicates contrast enhancement; CE-, no contrast enhancement; +, pathological; -, normal. and visual contrast enhancement between both sequences for each reader separately. Concordance between both raters was assessed using the intraclass correlation coefficient. Furthermore, the standardized contrast-to-noise ratio was compared using a 2sided Student $t$ test for paired samples. A value of $P<.05$ was considered statistically significant. Sensitivity and specificity were calculated and compared between sequences.

\section{RESULTS}

The diagnosis of acute $\mathrm{ON}$ was established by the treating neurologists for 30 of the 51 patients. Of these 30 patients with the final diagnosis of ON, 7 were known to have MS, ON was a first manifestation of MS in 9 patients, ON was a clinically isolated syndrome in 12 patients, and a neuromyelitis optica spectrum disorder was seen in the remaining 2 cases. VEP were available for 18 patients, with 14 exhibiting a pathologic pattern. Furthermore, OCT was available for 19 patients, with 5 showing a pattern of acute $\mathrm{ON}$ and 6 showing a pattern of atrophy.

Two discrepant lesion counts were found between readers (reader one, 21 lesions; reader two, 23 lesions), with reader 2 counting 2 artifacts as contrast-enhancing lesions in both sequences. In the consensus reading of both neuroradiologists, 21 patients showed at least 1 contrast-enhancing lesion in the optic nerve (unilateral, $n=20$; bilateral, $n=1$ ). In the case of bilateral contrast-enhancing lesions, analyses of only 1 optic nerve (with the longer lesion) were included in the statistics.

One patient showed signal alterations in DIR, FS T2WI, and FLAIR sequences but no contrast enhancement. Nine patients showed a T2-/DIR-hyperintense signal in the contralateral optic nerve without contrast enhancement and without recent clinical symptoms. The contrast-enhancing lesions were intraorbital $(n=$ 13), extraorbital intracanalicular $(n=4)$, extraorbital intracranial $(n=1)$, or intra- and extraorbital $(n=3)$. None of the contrastenhancing lesions detected in contrast-enhanced T1-weighted Dixon sequences were missed in contrast-enhanced T1-weighted $\mathrm{BB}$ sequences and vice versa. All contrast-enhancing lesions in T1-weighted images had a corresponding T2-/DIR-hyperintense signal. Eight patients with diagnoses of acute ON but no contrast-enhancing lesions also showed no signal alterations of the optic nerves in other sequences (DIR, FS T2WI, or FLAIR). VEP showed a normal pattern for 3 patients, and OCT findings were normal for 4 of these 9 patients without contrast-enhancing lesions. Contingency data with more information about diagnostics, sensitivity, and specificity are provided in Tables 2 and 3. Sensitivity (reader 1 and 2: 70\%) and specificity (reader 1, 100\%; and reader $2,90 \%$ ) were identical for both sequences regarding the final diagnoses.

Final diagnoses for the 21 patients not diagnosed with acute ON included other eye diseases not involving the optic nerve $(n=$ 4 ) or psychosomatic disorders $(n=3)$, or remained unclear $(n=$ $14)$; none of these patients' optic nerves showed a contrastenhancing lesion or a signal alteration in DIR or FS T2WI. Furthermore, none of these patients showed a pathologic pattern in VEP (available in 19/21 patients). OCT was available in 12/21 patients and showed a normal pattern in 11 patients; in 1 patient, it revealed a bilateral global atrophy of the peripapillary retinal 
Table 4: Results of the qualitative image evaluation ${ }^{\mathrm{a}}$

\begin{tabular}{|c|c|c|c|}
\hline Assessment & T1-Weighted BB (Mean) (95\% CI) & T1-Weighted Dixon (Mean) (95\% CI) & $P$ (Intrareader Comparison) \\
\hline \multicolumn{4}{|l|}{ Overall image quality } \\
\hline Reader 1 & $4.33 \pm 0.71(4.05-4.50)$ & $4.45 \pm 0.58(4.25-4.58)$ & .36 \\
\hline Reader 2 & $4.22 \pm 0.64(3.98-4.36)$ & $4.06 \pm 0.58(3.86-4.19)$ & .07 \\
\hline Mean & $4.27 \pm 0.68(4.05-4.41)$ & $4.25 \pm 0.61(4.13-4.40)$ & .81 \\
\hline \multicolumn{4}{|l|}{ Artifacts } \\
\hline Reader 1 & $4.25 \pm 0.69(3.98-4.41)$ & $4.57 \pm 0.57(4.36-4.71)$ & $.01^{\mathrm{b}}$ \\
\hline Reader 2 & $4.25 \pm 0.72(3.97-4.42)$ & $4.16 \pm 0.76(3.86-4.30)$ & .20 \\
\hline Mean & $4.25 \pm 0.70(4.03-4.39)$ & $4.36 \pm 0.70(4.17-4.49)$ & 0.17 \\
\hline \multicolumn{4}{|l|}{ Diagnostic confidence } \\
\hline Reader 1 & $4.22 \pm 1.00(3.70-4.43)$ & $4.24 \pm 0.74(3.96-4.39)$ & .90 \\
\hline Reader 2 & $4.41 \pm 0.80(4.08-4.59)$ & $3.96 \pm 0.94(3.55-4.14)$ & $<0.001^{\mathrm{b}}$ \\
\hline Mean & $4.31 \pm 0.91(3.98-4.49)$ & $4.10 \pm 0.85(3.86-4.24)$ & .04 \\
\hline \multicolumn{4}{|c|}{ Visual contrast enhancement } \\
\hline Reader 1 & $1.92 \pm 1.23(1.35-1.89)$ & $1.92 \pm 1.21(1.36-1.89)$ & 1.00 \\
\hline Reader 2 & $1.84 \pm 1.08(1.36-1.84)$ & $1.71 \pm 0.90(1.36-1.73)$ & $.007^{\mathrm{b}}$ \\
\hline Mean & $1.88 \pm 1.15(1.36-1.87)$ & $1.81 \pm 1.07(1.35-1.82)$ & 0.11 \\
\hline
\end{tabular}

${ }^{a}$ The results are listed separately per reader (reader $1+$ reader 2 ) and as mean values between the readers, given as mean values with SDs and $95 \%$ confidence intervals.

${ }^{\mathrm{b}}$ Significant differences between T1-weighted BB and T7-weighted Dixon $(P<.05)$.

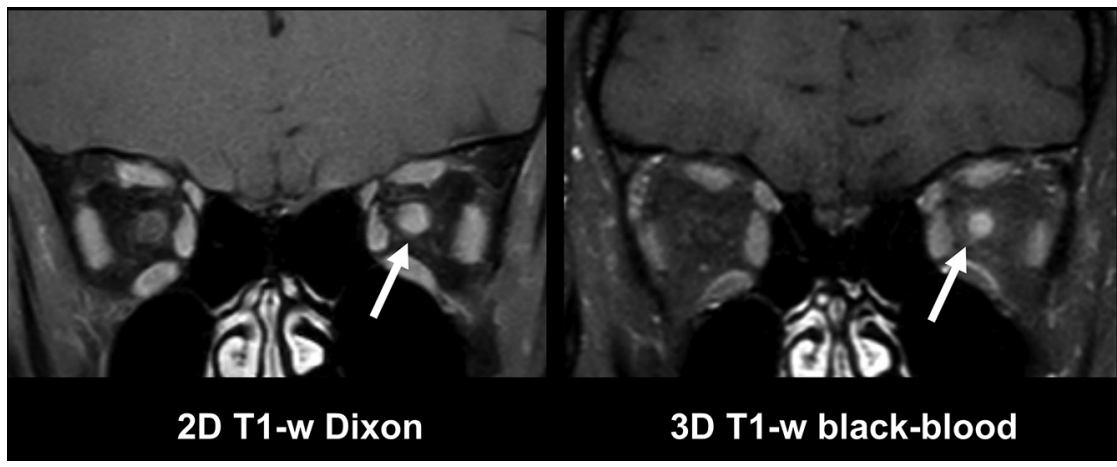

FIG 1. MR imaging of a 34-year-old female patient with symptoms of acute optic neuritis of the left eye with loss of vision and pain during eye movement for 3 days. Images of a contrastenhanced 2D T1-weighted Dixon sequence with coronal orientation (left) and a contrastenhanced 3D T1-weighted BB sequence with coronal reconstruction (right) are shown. Note a contrast-enhancing lesion in the left optic nerve in the intraorbital section with similarly high contrast in both sequences (arrows).

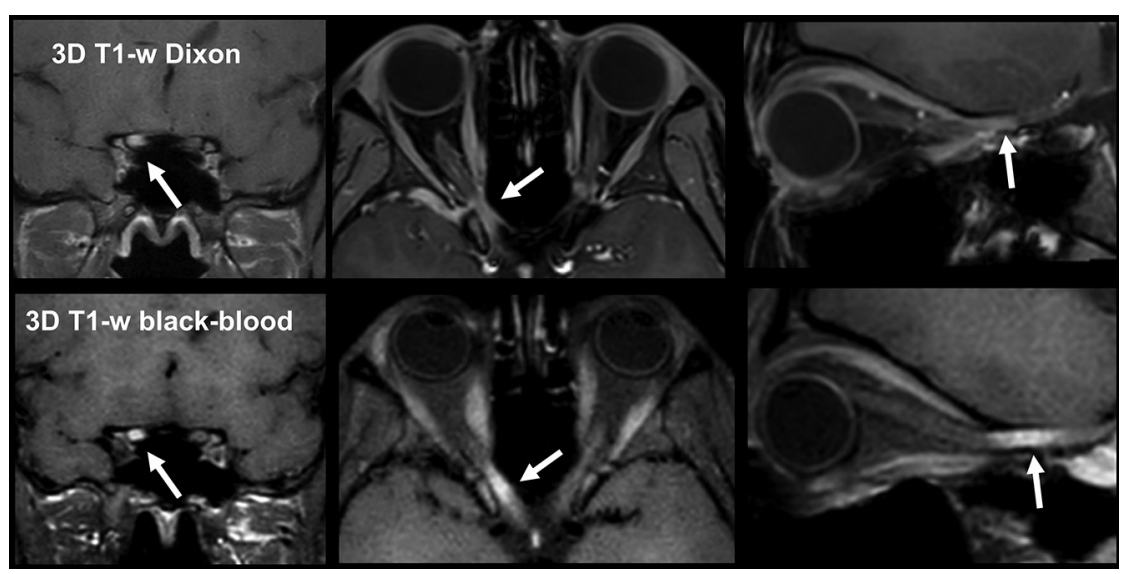

FIG 2. MR imaging of a 27-year-old female patient with symptoms of acute optic neuritis of the right eye with retrobulbar pain and loss of vision for 4 days. Images of a contrast-enhanced 3D T-weighted Dixon sequence are shown in the upper row, and contrast-enhanced 3D T-weighted BB images, in the lower row with coronal, axial, and sagittal reconstructions. Note the enhancing lesion in the right optic nerve in the extraorbital section (arrows) with improved contrast depiction in the contrast-enhanced 3D T-weighted BB sequence compared with the contrast-enhanced 3D T1-weighted Dixon imaging. nerve fiber layer and the macula, which was thought to be associated with another ocular disease.

The results of the qualitative image evaluation are listed in Table 4. Overall image quality, artifacts, diagnostic confidence, and visual contrast enhancement were rated by both readers similarly for $\mathrm{T} 1$-weighted $\mathrm{BB}$ and T1-weighted Dixon sequences, with a slight tendency toward better quality on T1-weighted BB sequences. The intraclass correlation coefficient was excellent and slightly higher when assessing T1-weighted BB compared with T1-weighted Dixon (0.923 versus 0.872).

There was no significant difference $(P=.27)$ between the standardized contrast-to-noise ratio of T1-weighted $\mathrm{BB}$ $(1.76 \pm 1.07)$ and T1-weighted Dixon (2.29 \pm 2.49). Examples of 2 patients are shown in Figs 1 and 2.

\section{DISCUSSION}

In this study, a contrast-enhanced 3D T1-weighted BB sequence successfully detected active contrast-enhancing lesions in the optic nerves in patients with ON. In particular, whole-brain contrast-enhanced 3D T1-weighted BB images showed high accuracy and qualitative/quantitative characteristics similar to those of dedicated orbital contrastenhanced T1-weighted Dixon sequences, with the advantage of shorter scan times. This finding is comparable with those of previous studies investigating 
different pathologies in the brain. ${ }^{13,15,16,24}$ Thus, the advantage of a fast 3D acquisition with sensitive contrast depiction of 3D T1weighted $\mathrm{BB}$ sequences also holds true in anatomically more complex regions such as the orbits and, in particular, the optic canal.

The results of our study are also in accordance with initial outcomes of the study of Sommer et al, ${ }^{13}$ describing the possibility of the assessment of the orbit using a T1-weighted modified volumetric isotropic turbo spin-echo acquisition to diagnose ON. However, they did not show any qualitative or quantitative data analysis.

Contrary to other studies ${ }^{8,12}$ that reported contrast enhancement of the optic nerve in acute $\mathrm{ON}$ in up to $94 \%$ of the cases, only $70 \%$ ( 21 of 30 patients) of the patients with acute ON showed contrast enhancement in our study. The contrast enhancement of the optic nerve in case of acute $\mathrm{ON}$ might depend on the severity of the inflammation or dimension of the affected optic nerve. Additionally, the high percentage of contrast enhancement in the above-mentioned study ${ }^{12}$ might depend on its strict inclusion and exclusion criteria (the study included patients within 20 days of visual loss, and patients were excluded if they had a history of ON of the affected eye and when vision did not return to normal). In our experience, the percentage of $70 \%$ contrast enhancement is closer to our referral clinical experience than the higher numbers of more selective studies. Furthermore, we included 1 patient who had already received high doses of IV prednisolone therapy before MR imaging; this patient showed a pathologic pattern in VEP but no contrast enhancement on MR imaging.

Recently, 1 study ${ }^{25}$ showed a potentially important role of a postcontrast FS FLAIR sequence demonstrating perioptic leptomeningeal contrast enhancement that might reflect an inflammatory process preceding or accompanying ON. We did not observe leptomeningeal enhancement on postcontrast T1-weighted BB sequences, but a dedicated study comparing postcontrast FLAIR sequences with postcontrast T1-weighted BB imaging for perioptic contrast enhancement might be of interest and clinical relevance.

One important advantage of 3D T1-weighted BB imaging is the short scan time. Because the acquisition of our whole-brain 3D T1weighted BB sequence takes only 1:30 (min:sec) at a 0.9-mm isotropic resolution and seems to lead to a higher detection rate of contrastenhancing MS lesions in the brain, ${ }^{13}$ it might replace standard T1weighted sequences such as MPRAGE and, importantly, dedicated sequences for the optic nerves, thus contributing to scan efficiency.

We are aware of several limitations of our study, including the relatively small sample size and retrospective study design. Furthermore, the readers could not be blinded to the type of sequences because they have characteristic appearances. In addition, the postcontrast T1-weighted sequences of this study were performed with different delays after intravenous administration of gadolinium-based contrast agents; on average, T1-weighted Dixon was performed 5 minutes later than contrast-enhanced T1-weighted BB. It is known that the sensitivity of postcontrast images increases with the length of delay after administration of a gadolinium-based contrast agent. ${ }^{26}$ Therefore, this difference might contribute to a bias but should not alter the main findings of this study in general.

\section{CONCLUSIONS}

Whole-brain contrast-enhanced 3D T1-weighted BB images of the optic nerves showed high accuracy and qualitative/quantitative characteristics similar to those of dedicated orbital contrastenhanced T1-weighted Dixon images to diagnose acute ON. Thus, if contrast-enhanced 3D T1-weighted $\mathrm{BB}$ images are routinely acquired for the detection of contrast-enhancing MS lesions in the brain, potentially, additional dedicated orbital sequences may not be needed, allowing shorter standard MR imaging protocols.

\section{ACKNOWLEDGMENTS}

The authors would like to thank Mrs Marianne SchoenbachEngmann for proofreading the manuscript.

Disclosures: Mark Mühlau-RELATED: Grant: research fund, Comments: German Federal Ministry for Education and Research, German Competence Network Multiple Sclerosis, grant No. 01GI1604A.* Claus Zimmer-RELATED: C. Zimmer disclosed no relevant relationships regarding activities related to the present article. UNRELATED: He has served on scientific advisory boards for Philips and Bayer Schering, serves as co-editor on the Advisory Board of Clinical Neuroradiology, has received speaker honoraria from Bayer Schering and Philips Healthcare; the institution has received research support and investigator fees for clinical studies from Biogen Idec, Quintiles Transnational, Merck Sharp \& Dome, Boehringer Ingelheim, Inventive Health Clinical UK Ltd, AdvanceCor Biotechnologie, BrainsGate, Pfizer, Bayer Schering, Novartis, Roche, Servier, Penumbra, WCT GmbH, Syngis, SSS International Clinical Research, PPD Germany GmbH, Worldwide Clinical Trials Ltd, phenox, Covidien, Actelion, Medivation, Medtronic, Harrison Clinical Research, Concentric, PharmTrace, Reverse Medical Corp, Premier Research Germany Ltd, Surpass Medical Ltd, GlaxoSmithKline, AXON Neuroscience, Bristol-Myers Squibb, Genentech, Acandis, Eisai, NeuroRx, Italfarmaco, BioClinica, MIAC Analytics, and IXICO.* No patents issued and pending. Jan S. Kirschke-UNRELATED: Grants/ Grants Pending: Deutsche Forschungsgemeinschaft, European Research Council, Nvidia Corporation*; Payment for Lectures Including Service on Speakers Bureaus: Philips Healthcare. *Money paid to the institution.

\section{REFERENCES}

1. Francis DA, Compston DA, Batchelor JR, et al. A reassessment of the risk of multiple sclerosis developing in patients with optic neuritis after extended follow-up. J Neurol Neurosurg Psychiatry 1987;50:75865 CrossRef Medline

2. Rizzo JF 3rd, Lessell S. Risk of developing multiple sclerosis after uncomplicated optic neuritis: a long-term prospective study. Neurology 1988;38:185-90 CrossRef Medline

3. Hickman SJ, Dalton CM, Miller DH, et al. Management of acute optic neuritis. Lancet 2002;360:1953-62 CrossRef Medline

4. Toosy AT, Mason DF, Miller DH. Optic neuritis. Lancet Neurol 2014;13:83-99 CrossRef Medline

5. Wikstrom J, Poser S, Ritter G. Optic neuritis as an initial symptom in multiple sclerosis. Acta Neurol Scand 1980;61:178-85 CrossRef Medline

6. Filippi M, Rocca MA, Ciccarelli O, et al; MAGNIMS Study Group. MRI criteria for the diagnosis of multiple sclerosis: MAGNIMS consensus guidelines. Lancet Neurol 2016;15:292-303 CrossRef Medline

7. Thompson AJ, Banwell BL, Barkhof F, et al. Diagnosis of multiple sclerosis: 2017 revisions of the McDonald criteria. Lancet Neurol 2018;17:162-73 CrossRef Medline

8. Petzold A, Wattjes MP, Costello F, et al. The investigation of acute optic neuritis: a review and proposed protocol. Nat Rev Neurol 2014;10:447-58 CrossRef Medline

9. Hadhoum N, Hodel J, Defoort-Dhellemmes S, et al. Length of optic nerve double inversion recovery hypersignal is associated with retinal axonal loss. Mult Scler 2016;22:649-58 CrossRef Medline

10. Hodel J, Outteryck O, Bocher AL, et al. Comparison of 3D double inversion recovery and 2D STIR FLAIR MR sequences for the imaging of optic neuritis: pilot study. Eur Radiol 2014;24:3069-75 CrossRef Medline

11. Riederer I, Muhlau M, Hoshi MM, et al. Detecting optic nerve lesions in clinically isolated syndrome and multiple sclerosis: double-inversion recovery magnetic resonance imaging in comparison 
with visually evoked potentials. J Neurol 2019;266:148-56 CrossRef Medline

12. Kupersmith MJ, Alban T, Zeiffer B, et al. Contrast-enhanced MRI in acute optic neuritis: relationship to visual performance. Brain 2002;125:812-22 CrossRef Medline

13. Sommer NN, Saam T, Coppenrath E, et al. Multiple sclerosis: improved detection of active cerebral lesions with 3-dimensional T1 black-blood magnetic resonance imaging compared with conventional 3-dimensional T1 GRE imaging. Invest Radiol 2018;53:13-19 CrossRef Medline

14. Park J, Kim J, Yoo E, et al. Detection of small metastatic brain tumors: comparison of 3D contrast-enhanced whole-brain blackblood imaging and MP-RAGE imaging. Invest Radiol 2012;47:13641 CrossRef Medline

15. Yoneyama M, Nakamura M, Tabuchi T, et al. Whole-brain blackblood imaging with magnetization-transfer prepared spin echolike contrast: a novel sequence for contrast-enhanced brain metastasis screening at 3T. Radiol Phys Technol 2013;6:431-36 CrossRef Medline

16. Kammer NN, Coppenrath E, Treitl KM, et al. Comparison of contrast-enhanced modified T1-weighted 3D TSE black-blood and 3D MP-RAGE sequences for the detection of cerebral metastases and brain tumours. Eur Radiol 2016;26:1818-25 CrossRef Medline

17. Treitl KM, Treitl M, Kooijman-Kurfuerst H, et al. Three-dimensional black-blood T1-weighted turbo spin-echo techniques for the diagnosis of deep vein thrombosis in comparison with contrast enhanced magnetic resonance imaging: a pilot study. Invest Radiol 2015;50:401-08 CrossRef Medline

18. Edelman RR, Chien D, Kim D. Fast selective black-blood MR imaging. Radiology 1991;181:655-60 CrossRef Medline
19. Edelman RR, Mattle HP, Wallner B, et al. Extracranial carotid arteries: evaluation with "black-blood" MR angiography. Radiology 1990;177:45-50 CrossRef Medline

20. Treitl KM, Maurus S, Sommer NN, et al. 3D-black-blood 3T-MRI for the diagnosis of thoracic large vessel vasculitis: a feasibility study. Eur Radiol 2017;27:2119-28 CrossRef Medline

21. Sommer NN, Treitl KM, Coppenrath E, et al. Three-dimensional high-resolution black-blood magnetic resonance imaging for detection of arteritic anterior ischemic optic neuropathy in patients with giant cell arteritis. Invest Radiol 2018;53:698-704 CrossRef Medline

22. Riederer I, Karampinos DC, Settles M, et al. Double inversion recovery sequence of the cervical spinal cord in multiple sclerosis and related inflammatory diseases. AJNR Am J Neuroradiol 2015;36:21925 CrossRef Medline

23. Edelstein WA, Glover GH, Hardy CJ, et al. The intrinsic signal-to-noise ratio in NMR imaging. Magn Reson Med 1986;3:604-18 CrossRef Medline

24. Park J, Kim EY. Contrast-enhanced, three-dimensional, wholebrain, black-blood imaging: application to small brain metastases. Magn Reson Med 2010;63:553-61 CrossRef Medline

25. Pino-Lopez L, Wenz H, Bohme J, et al. Contrast-enhanced fatsuppressed FLAIR for the characterization of leptomeningeal inflammation in optic neuritis. Mult Scler 2019;25:792-800 CrossRef Medline

26. Uysal E, Erturk SM, Yildirim H, et al. Sensitivity of immediate and delayed gadolinium enhanced MRI after injection of $0.5 \mathrm{M}$ and 1.0 $\mathrm{M}$ gadolinium chelates for detecting multiple sclerosis lesions. $A J R$ Am J Roentgenol 2007;188:697-702 CrossRef Medline 